\title{
Maintenance decision method considering inspection of mining equipment
}

\author{
Xian Gang $\mathrm{Cao}^{1}$, Meng Yuan Zhang ${ }^{1,2, *}$, Yu Rong Gong ${ }^{1}$, Xiao Lin $\mathrm{Jia}^{2}$, and Rui Yuan Zhang ${ }^{1}$ \\ ${ }^{1}$ School of Mechanical Engineering, Xi'an University of Science and Technology 710054, PR China \\ ${ }^{2}$ School of Mechanical and Aerospace Engineering, Brunel University London, London UB8 3PH, UK
}

Received: 12 November 2020 / Accepted: 8 July 2021

\begin{abstract}
In coal mining industry, equipment safety and reliability during operation are essential in the production process. In recent years, along with the continuous improvement of intelligent coal mining equipment, reliable production requirements have simultaneously increased. Therefore, this article aims to study the maintenance decision making method that considers the safety inspection of a fully mechanized mining equipment group. The goal is to establish a maintenance decision making model that considers the safety overhaul of fully mechanized mining equipment group in order to minimize the total maintenance, production costs and safety risks. Firstly, the regular safety inspection procedure and the maintenance process were introduced. Then, a model guideline method that considers a fully mechanized mining equipment group's safety inspection was established. Finally, through the comparison of algorithms and maintenance strategies, it is proved that the maintenance decision optimization method proposed in this paper is of great significance for improving equipment reliability, improving comprehensive operation rate, reducing maintenance costs, and ensuring coal mine safety production.
\end{abstract}

Keywords: Comprehensive mining equipment group / maintenance decision making / cost / safety / genetic algorithm

\section{Introduction}

The failure of coal mining equipment is one of the most influential factors that restrict the smooth running during the coal mining operation. When maintenance is carried out improperly, it can easily affect the operation of the entire system, causing unpredictable and immeasurable economic losses and accidents regarding safety. According to studies and statistics, a coal mine which produces 10 million tons of coal annually can lose at least 10 million Chinese yuan during a single day of shutdown due to equipment failure [1]. Numerous coal mines have experienced not only a vast number of financial losses, but safety casualties at the same time. Therefore, improving the reliability of mining equipment is essential for the safety and sustainability of coal mines production [2].

In the meantime, the Maintenance Decision Making Model is based on the perspective of the system and can be divided into Decision Making Model with a single device as the research objective, or a model with an entire production system consist of multiple devices as the research subject

* Corresponding author: 582060393@qq.com matter. The maintenance decision model for a single device is relatively simple and have many public sources in this area of research. It focuses on the reliability of a single component or multiple components of a single device to calculate the maintenance cost, availability, maintenance time, and equipment under different constraint conditions [3]. The multi device maintenance needs to consider the correlation between all the devices and the research process, which is much more complicated. There are three types of correlations commonly found in multi equipment production system: economic correlation, structural correlation, and random correlation [4].

Lin et al. have established a multi objective decision making model based on condition maintenance in the process of evaluating the reliability of aircraft structure [5]. The model focused on minimizing maintenance costs and maximizing fleet availability. This decision making system (MDMS) proved the model's effectiveness through experiments. Eruguz et al. established a maintenance decision making model based on the number of active units, the degradation rate of active units and standby units, maintenance costs, and economic dependence types by describing the degradation of equipment service age. The model decides which unit to maintain based on the different 


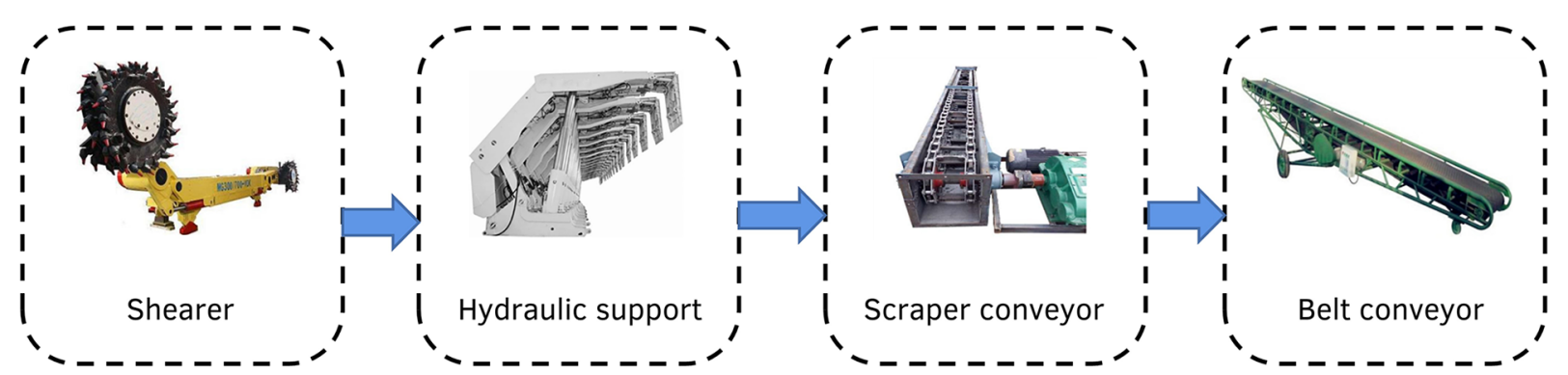

Fig. 1. Simplified layout of fully mechanized coal mining equipment group (In this article, Hydraulic Support Group is referred to as just Hydraulic Support).

degradation rates of the in use and standby units. Experiments have proved that the cost increase caused by the previous maintenance strategy is as high as $27 \%$, and the joint maintenance optimization is more valuable [6]. When domestic Chinese and foreign researchers establish maintenance decision making models, they mainly take maintenance cost, maintenance time, system operation risk as decision making goals [7], aiming to ensure equipment reliability and improve production system operating rate.

This paper establishes a maintenance decision making model that considers the safety overhaul of the fully mechanized mining equipment group with the goal of minimizing the total cost of maintenance and production. The experimental verification and comparison of maintenance strategies prove that the maintenance decision optimization method proposed in this paper can effectively improve the equipment reliability. Performance and comprehensive operating rate, and reduce maintenance costs, thus improve the coal industry's sustainable development.

\section{Problem description and hypothesis}

The underground coal mining environment is harsh and the mining system is vast and complex, which is prone to hidden dangers during production [8]. Therefore, coal mining production's safety status needs regular, professional, prominent, and focused inspection and supervision. China always puts the safety of coal mining production first, and consistently provides consistent scientific analysis and professional management on coal mining companies' safety level. The safety inspection and repair of coal mining equipment are among the most critical factors to ensure safe production [9].

During the coal mining process, simply judging whether maintenance is required based on equipment failure rate cannot thoroughly eliminate the safety risks caused by potential equipment failures [10]. Therefore, further inspections based on the opportunity maintenance strategy plays a significant role in ensuring the production safety of coal mines.

In this paper, the following hypothesis is made in the research on the optimization method of maintenance decision making considering the safety overhaul of fully mechanized mining equipment group:
- The simplified layout of fully mechanized coal mining equipment group is as shown in Figure 1.

- The fully mechanized mining equipment group can fail at any time during production, and the performance of the equipment will decline over time, therefore the failure rate will increase accordingly. The failure rate of recording equipment $\mathrm{i}$ is $F R_{i}(t)$ and $F R_{i}(t)$ obeys the Weibull distribution [11].

- During the maintenance plan period, the overhaul of the fully mechanized mining equipment group will be automatically completed if the equipment needs to be moved or reversed. Therefore, this article does not consider the cost of overhaul during relocation.

- The equipment failure rate will also increase during the production process as the machines are being used repetitively. Although the maintenance cannot repair the equipment to the same level that brand new equipment has but taking certain maintenance activities simultaneously with the production process will still reduce the equipment failure rate and improve the equipment's performance.

- Divide the maintenance plan period into maintenance intervals and judge the number of opportunities for maintenance in each interval.

This article only considers the safety overhaul of the fully mechanized coal mining equipment group. It does not consider the impact of the external environment on the internal safety of the coal mine. During the maintenance plan period, the overhaul of the fully mechanized mining equipment group will be automatically completed if the equipment needs to be moved or reversed. Therefore, this article does not consider the cost of overhaul during relocation. The safety inspection of the fully mechanized coal mining equipment group will not affect the production system's regular operation.

\section{Model establishment and potential solutions}

\subsection{Maintenance decision model of fully mechanized mining equipment group}

\subsubsection{Maintenance decision model}

When establishing the maintenance decision making model for the fully mechanized mining equipment group, the 


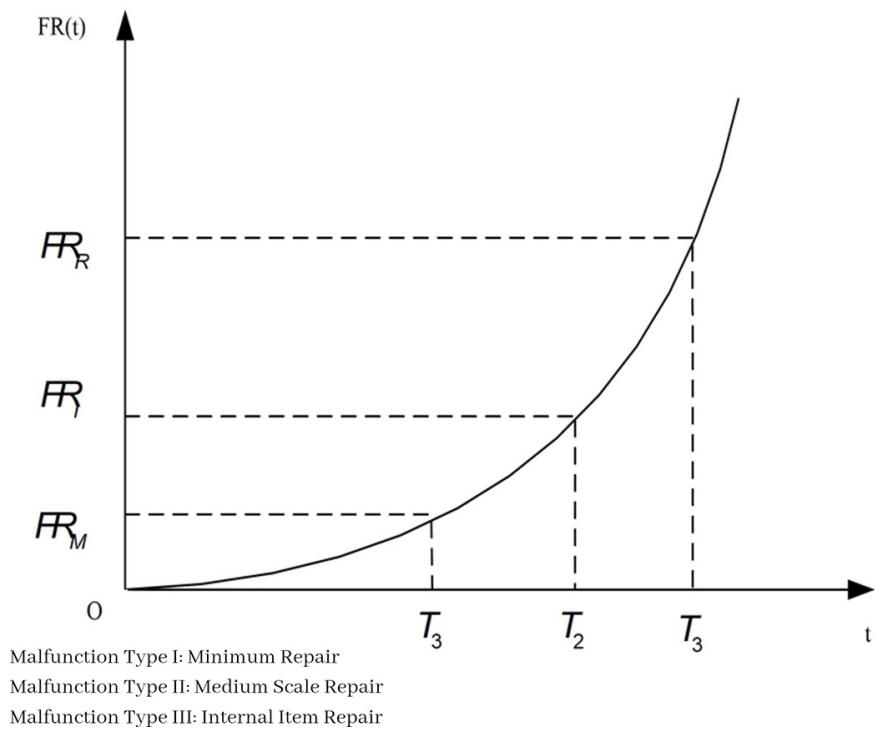

Fig. 2. Equipment degradation trend chart.

primary consideration is the equipment group's opportunity maintenance cost, sudden failure and minor repair, the cost of downtime loss, and the depreciation loss cost after medium repair/item repair. The equipment deterioration trend is shown in Figure 2. The specific descriptions of minor maintenance, medium repairs/item repairs are as follows.

When the failure rate rises from 0 to $F R_{M}(t)$, it is a minor fault, and the maintenance method of minimum repair (minor repair) is adopted. Minor faults are mainly caused by the damage of a specific part of the equipment. This kind of fault only considers the maintenance of a single part and should not change the equipment's failure rate. When the failure rate rises from $F R_{M}(t)$ to $F R_{I}(t)$, it is a medium level failure, and the maintenance method is medium repair. This maintenance method involves disassembling and repairing the key components that failed in the fully mechanized mining equipment group. This type of repair mainly includes replacement of complete sets of parts, and cleaning of parts, etc. When the failure rate rises from $F R_{I}(t)$ to $F R_{R}(t)$, It is a more serious failure, and the maintenance method of internal item repair is adopted. This failure is mainly caused by the overall aging or fatigue of the equipment.

(1) Opportunity maintenance cost:

$$
C_{m}=\sum_{j=1}^{J} \sum_{i=1}^{N}\left(c_{i}^{p} m_{i j}+c_{i}^{r} r_{i j}+c_{i}^{f} l_{i j}\right) .
$$

In the equation, $C_{m}$ represents the opportunity maintenance cost, $c_{i}^{p}$ is medium-level repair and maintenance costs for equipment, $c_{i}^{r}$ item repair and maintenance costs for equipment, $c_{i}^{f}$ is maintenance cost for a single minor repair of the equipment, $m_{i j}$ is for the equipment to be repaired at the end of the maintenance interval, $r_{i j}$ is decision variables for equipment repair at the end of the maintenance interval, $l_{i j}$ is a decision variable for equipment minor repair at the end of the maintenance interval, $l_{i j}$ is a decision variable for equipment minor repair at the end of the maintenance interval.

(2) Cost of minor repairs during sudden failures:

If the number of sudden failures occurred with equipment $\mathrm{i}$ in maintenance interval $j$ is $n_{i j}$. The expected number of failures is $E\left(n_{i j}\right)$. Since each device obeys the Weibull distribution, according to the reliability theory, the following equation was deducted:

$$
\begin{aligned}
E\left(n_{i j}\right) & =\int_{x_{i j}}^{x_{i j}^{\prime}} F R(t) d t=\int_{x_{i j}}^{x_{i j}^{\prime}} \frac{b_{i}}{a_{i}} \cdot\left(\frac{t}{a_{i}}\right)^{b_{i}-1} d t \\
& =\frac{1}{a_{i}}\left[\left(\frac{x_{i j}^{\prime}}{a_{i}}\right)^{b_{i}}-\left(\frac{x_{i j}}{a_{i}}\right)^{b_{i}}\right] .
\end{aligned}
$$

Therefore, if equipment $i$ suffered sudden failure in maintenance interval $j$ and need minor repairment, the cost can be represented as:

$$
\frac{c_{i}^{f}}{a_{i}}\left[\left(\frac{x_{i j}^{\prime}}{a_{i}}\right)^{b_{i}}-\left(\frac{x_{i j}}{a_{i}}\right)^{b_{i}}\right] .
$$

The minor repair cost of sudden equipment failure during the entire maintenance plan period can be expressed as [12]:

$$
C_{d}=\sum_{j=1}^{J} \sum_{i=4}^{N}\left\{\frac{c_{i}^{f}}{a_{i}}\left[\left(\frac{x_{i j}^{\prime}}{a_{i}}\right)^{b_{i}}-\left(\frac{x_{i j}}{a_{i}}\right)^{b_{i}}\right]\right\}
$$

In the equation, $C_{d}$ is the total cost of minor repairment for the equipment group, $c_{i}^{f}$ is the single minor repairment cost for equipment, $a_{i}$ is the scale parameter of the device, $b_{i}$ is the shape parameter of the device $i$, and $a>0, b>0, x_{i j}$ and $x_{i j}^{\prime}$ each is the functioning life of $i$ when maintenance interval $j$ start and ends.

If the equipment $i$ is maintained during maintenance interval $j$, the impact on the effective service life of the equipment is:

$$
x_{i j+1}=\beta \cdot x_{i j}^{\prime}, i=1, \ldots N, j=1, \ldots J-1
$$

If the equipment $i$ is not maintained at the end of maintenance interval $j$, the sufficient service age of the equipment remains unchanged.

$$
x_{i j+1}=x_{i j}^{\prime}, i=1, \ldots N, j=1, \ldots J-1 .
$$

(3) Downtime loss:

The downtime loss is mainly due to the production loss caused by equipment shutdown and maintenance. In coal mine production, minor repairs will not cause production downtime. Therefore, the production downtime caused by 
maintenance $\left(\tau^{d}\right)$ can be expressed as:

$$
\begin{aligned}
\tau^{d}= & \sum_{j=1}^{J}\left\{1-\prod_{i=1}^{N}\left[1-\left(m_{i j}+r_{i j}\right)\right]\right. \\
& \left.+\sum_{i=1}^{N} \frac{1}{a_{i}}\left[\left(\frac{x_{i j}^{\prime}}{a_{i}}\right)^{b_{i}}-\left(\frac{x_{i j}}{a_{i}}\right)^{b_{i}}\right]\right\} .
\end{aligned}
$$

Therefore, the total downtime loss cost of the equipment group during the production cycle $(J)$ can be expressed as:

$$
C_{q}=V \cdot \tau^{d}
$$

In the equation, $V$ is the production benefit per unit time when the equipment group have not been paused.

(4) The cost of depreciation loss after medium scale/ item repair of equipment.

When Tambe [13] completed the calculation of equipment depreciation loss cost, they proposed the loss of equipment caused by replacement, when service age is $t^{\prime}$, the cost of replacing old equipment is represented by $r v_{i}\left(t^{\prime}\right)$. The equation is [12]:

$$
r v_{i}\left(t^{\prime}\right)=c_{i} \cdot \frac{\frac{1}{R_{i}\left(t^{\prime}\right)} \int_{t^{\prime}}^{\infty} R_{i}(t) d t}{\int_{0}^{\infty} R_{i}(t)}
$$

In the equation, $c_{i}$ is the single replacement cost for equipment $i, R_{i}(t)$ is the reliability of equipment $i$ at time $t$.

This article considers that when medium scale repair or item repair is carried out on the equipment, it is to replace some parts of the original equipment. Therefore, the depreciation loss cost to repair the equipment can be expressed as:

$$
\begin{aligned}
& c_{p}\left(t^{\prime}\right)=r v_{i}\left(t^{\prime}\right) \cdot \frac{c_{i}^{p}}{c_{i}} \\
& c_{r}\left(t^{\prime}\right)=r v_{i}\left(t^{\prime}\right) \cdot \frac{c_{i}^{r}}{c_{i}} .
\end{aligned}
$$

In the formulas, $c_{p}\left(t^{\prime}\right)$ is the depreciation loss cost of the equipment during the service age $t^{\prime} ; c_{r}\left(t^{\prime}\right)$ is the depreciation loss cost of the equipment repair during the service age $t^{\prime}$.

Therefore, the maintenance decision model of the coal mine comprehensive mining equipment group is as follows:

$$
\begin{aligned}
C_{T 1}= & \sum_{j=1}^{J} \sum_{i=1}^{N}\left\{c_{i}^{f}\left[l_{i j}+\frac{1}{a_{i}}\left(\frac{x_{i j}^{\prime}}{a_{i}}\right)^{b_{i}}-\left(\frac{x_{i j}}{a_{i}}\right)^{b_{i}}\right]\right. \\
& \left.+c_{i}^{p}\left(m_{i j}+c_{p}\left(x_{i j}^{\prime}\right)\right)+c_{i}^{r}\left(r_{i j}+c_{r}\left(x_{i j}^{\prime}\right)\right)\right\}+V \cdot \tau^{d} .
\end{aligned}
$$

\subsubsection{Safety inspection model}

It is possible to discover potential failures during regular safety inspections of the fully mechanized coal mining equipment group. During the planned maintenance period, one of two scenarios of safety inspection and repair of equipment may occur, and the corresponding cost can be expressed as:

(1) During the safety inspection and repair of the equipment, potential failures of the equipment were discovered, so the equipment was maintained with an opportunity maintenance strategy. The cost of equipment safety maintenance can be expressed as:

$$
C_{s}=\sum_{j=1}^{J}\left\{\left[\sum_{i=1}^{N}\left(c_{i}^{p} m_{i j}+c_{i}^{r} r_{i j}+c_{i}^{f} l_{i j}\right)+V \cdot \tau^{d}+C_{f}\right] P_{m}\left(t_{j}\right)\right\} .
$$

In the equation, $C_{s}$ represents the total cost of safety inspection under conditions of comprehensive mining work plan period $t$ and carried out over interval time period $J . j$ represents $J$ amount of maintenance interval. $P_{m}\left(t_{j}\right)$ is the probability of needing maintenance when time is $t_{j}$.

(2) During the safety inspection of the equipment, no potential faults were found, so there is no need to update the equipment failure rate. Then there is only inspection fee at this time, which can be expressed as:

$$
C_{n}=J \cdot C_{f} \cdot P_{n}\left(t_{j}\right)
$$

In this equation, $C_{n}$ represents the inspection cost when there is no safety hazard during equipment safety inspection. $P_{n}\left(t_{j}\right)$ is the probability that only security checks are performed at time $t_{j}$.

To summarize the above, we can conclude that the inspection cost before a certain time (represented by $t$ ) during the maintenance schedule is represented by this equation:

$$
\begin{aligned}
C_{T 2}= & \sum_{j=1}^{J}\left\{\left[\sum_{i=1}^{N}\left(c_{i}^{p} m_{i j}+c_{i}^{r} r_{i j}+c_{i}^{f} l_{i j}\right)\right.\right. \\
& \left.\left.+V \cdot \tau^{d}+C_{f}\right] P_{m}\left(t_{j}\right)\right\}+J C_{f} P_{n j}(t) .
\end{aligned}
$$

3.1.3 The maintenance decision model considering the safety inspection of fully mechanized mining equipment group

Finally, a maintenance decision-making model considering the safety overhaul of fully mechanized mining equipment group with the goal of minimizing the total cost of maintenance and production is established, as 


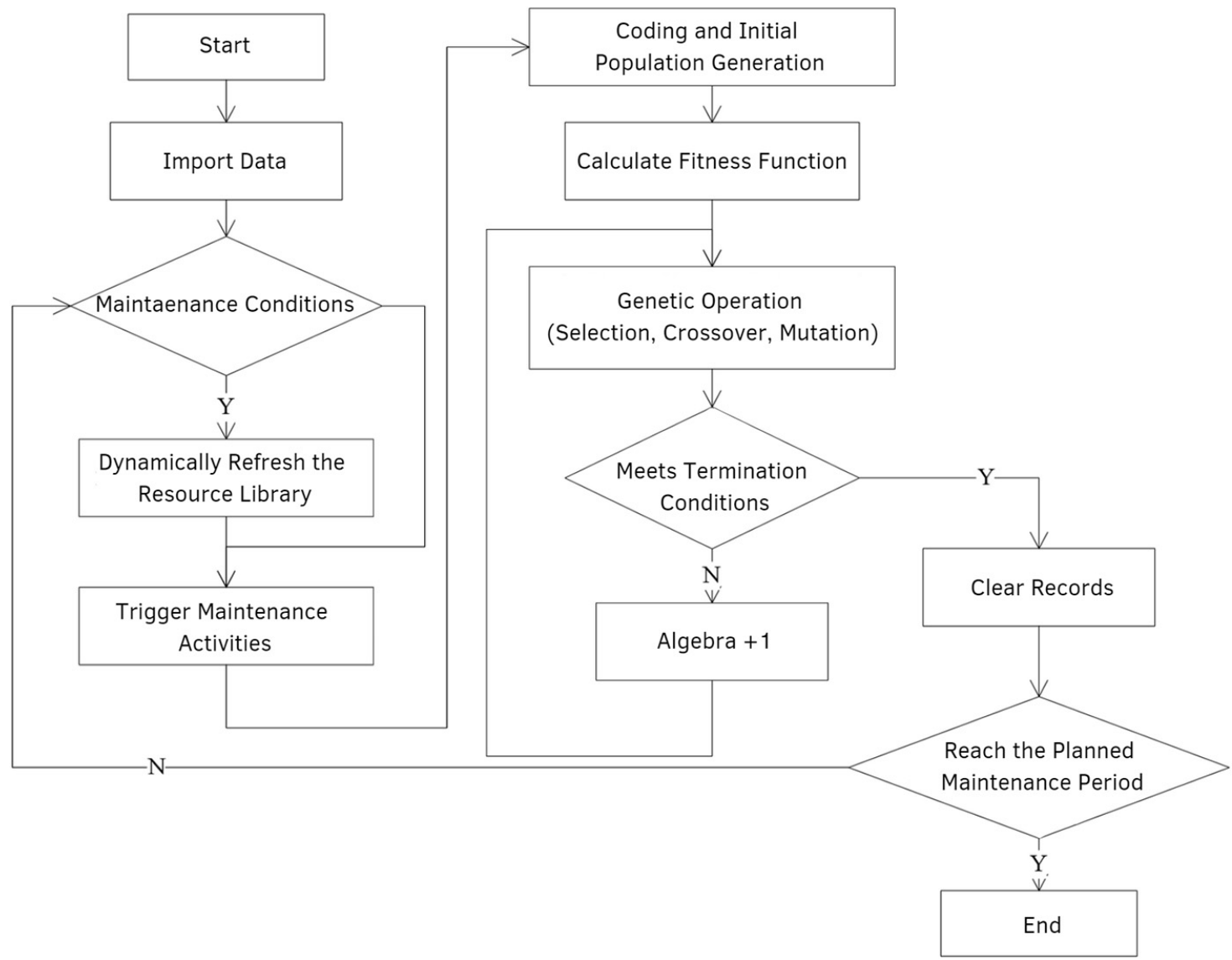

Fig. 3. Flow chart of equipment maintenance decision optimization.

shown in equation:

$$
\begin{aligned}
& \min C_{T}=C_{T 1}+C_{T 2} \\
& =\sum_{j=1}^{J} \sum_{i=1}^{N}\left\{c _ { i } ^ { f } \left[l_{i j}+\frac{1}{a_{i}}\left(\left(\frac{x^{\prime} i j}{a_{i}}\right)^{b_{i}}-\left(\frac{x_{i j}}{a_{i}}\right)^{b_{i}}\right]\right.\right. \\
& \left.+c_{i}^{p}\left(m_{i j}+c_{p}\left(x_{i j}^{\prime}\right)\right)+c_{i}^{r}\left(r_{i j}+c_{r}\left(x_{i j}^{\prime}\right)\right)\right\}+V \cdot \tau^{d} \\
& +\sum_{j=1}^{J}\left\{\left[\sum_{i=1}^{N}\left(c_{i}^{p} m_{i j}+c_{i}^{r} r_{i j}+c_{i}^{f} l_{i j}\right)+V \cdot \tau^{d}+C_{f}\right] P_{m}\left(t_{j}\right)\right\} \\
& +J C_{f} P_{n j}(t) .
\end{aligned}
$$

\subsection{Model solution}

The genetic algorithm is used to solve and optimize the maintenance decision model that considers the safety overhaul of the fully mechanized mining equipment group in this segment. The selection of the genetic algorithm parameters in this paper is based on previous algorithm design experience, and many accumulated experiments, including the number of genetic iterations and population, Selection operator, crossover operator and mutation operator. The maximum number of iterations is 200 .
The lowest maintenance cost and reasonable maintenance decision making arrangement can be calculated through the iterative optimisation of the algorithm. The solution process is shown in Figure 3:

- Import the basic data of the model in this paper, mainly including the number of fully mechanized mining equipment, the maintenance cost of different maintenance methods, the maintenance time of different maintenance methods, the law of performance decline of different equipment, and the maintenance thresholds of different maintenance methods.

- Consider the opportunity maintenance strategy to determine whether the maintenance conditions are met (item repair threshold, safety inspection).

- Encode the different maintenance methods of maintenance decision-making, generate the initial population, and calculate the fitness function.

\subsection{Genetic algorithm design}

Genetic Algorithms (GA) is an adaptive algorithm that simulates the evolutionary process of biological "survival of the fittest". The evolution process of the algorithm mainly uses the "selection, crossover, mutation" of the genetic operator to perform genetic operations, and finally converges to the adaptive group, so as to obtain the most satisfactory solution to the required problem. 


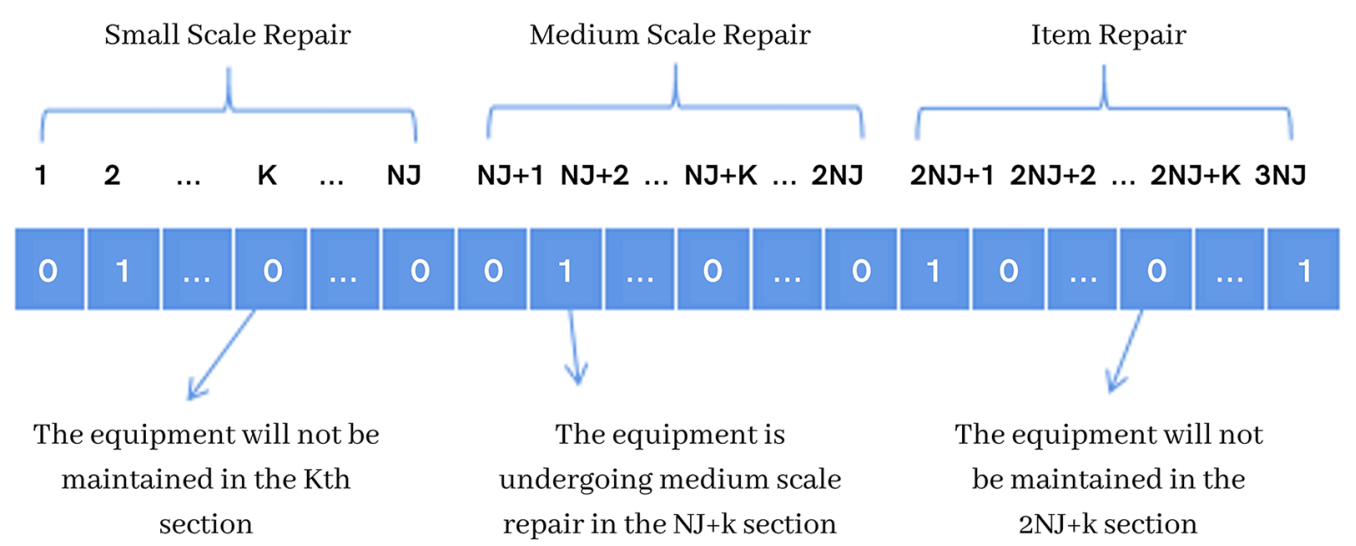

Fig. 4. Coding diagram.

(1) Chromosome coding

The three maintenance methods (minor repair, intermediate repair, or item repair) in this article are encoded using binary encoding, that is, $0 / 1$ variable, as shown in Figure 4 . The total length of the chromosome is $3 N \cdot J$, the first $N \cdot J$ genes represent minor equipment repairs, the middle $N \cdot J$ genes represent mid-equipment repairs, and the last $N \cdot J$ genes represent item repairs.

(2) Initial population generation

The choice of population size will affect the performance of a genetic algorithm. If the population is too small, the optimization efficiency will be poor. Which means a feasible solution will not be obtained; if the population is too large, the amount of calculation will increase during the optimization process, and the calculation speed will be significantly reduced [14]. Generally, the population size is selected between 40 and 100. In this chapter, the population size is chosen as 100 . Initial populations are randomly generated, representing different maintenance methods in each maintenance interval during the maintenance plan. If the $K$ th gene, $N \cdot J+k$ th and $2 N \cdot J+k$ th genes are all 1 , it means that minor repairs, intermediate repairs, and item repairs on the same equipment violate the production system constraints. Currently, the Kth, $N \cdot J+k$ th and $2 N \cdot J+k$ th genes are randomly selected. One of them is 1 , and the rest are 0 .

(3) Evaluation of individual fitness function

For this article, the maintenance decision-making model for the safety overhaul of a fully mechanized mining equipment group is taken as the fitness function [15]. The objective function is mainly to minimize the maintenance cost. Therefore, this article takes the reciprocation of the objective function as the fitness function. The value of the objective function and the fitness function is transformed, as shown in equation (18).

$$
\text { See equation (17) below. }
$$

(4) Selection operator

The selection operator in genetic operations mainly selects effective individuals for iterative operations, which improves global convergence. This article uses mostly the classic roulette method for selection operations; that is, individuals are selected according to formula (19). Every two individuals form a group, one as the parent and one as the mother to replicate.

$$
P_{i}=F_{i} / \sum_{i=1}^{N} F_{i} .
$$

\section{(5) Crossover operator}

The crossover operation is mainly to design the crossover position and crossover method of two crossover individuals. In this paper, the selection order of genetic operators is crossed. As shown in Figure 5 as an example, the specific operation steps for sequence crossing are as follows:

- Gene fragments of two chromosomes are randomly generated in the chromosomes of parent 1 and parent 2 . - Delete the gene fragments that have not been exchanged in the parent and save the exchanged genes.

- Insert the deleted genes into the gene fragments in reverse order.

$$
\begin{aligned}
& \min f(x)=\frac{1}{C_{T 1}+C_{T 2}} \\
& =1 / \sum_{j=1}^{J} \sum_{n=1}^{N}\left\{c_{i}^{f} \lambda_{i}\left[\left(x_{i j}^{\prime}\right)^{\beta_{i}}-\left(x_{i j}\right)^{\beta_{i}}+c_{i}^{p}\left(m_{i j}+C_{p}\left(x_{i j}^{\prime}\right)+C_{r}\left(x_{i j}^{\prime}\right)\right)\right\}\right. \\
& +C_{q}+\sum_{j=1}^{J}\left\{\left[j C_{f}+C_{e}+C(J, t-y)\right] P_{m}\left(t_{j}\right)\right\}+J C_{f} P_{n j}(t)
\end{aligned}
$$




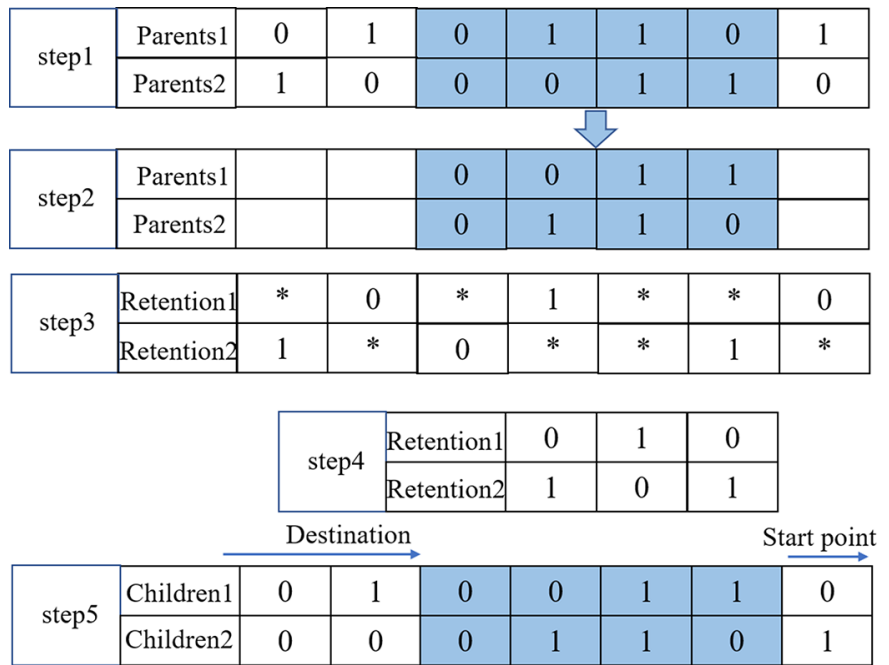

Fig. 5. Schematic diagram of cross operation.

\begin{tabular}{|c|c|c|c|c|c|c|c|c|c|}
\hline Parents & 0 & 1 & 1 & 0 & 1 & 0 & 1 & 1 & 1 \\
\hline Children & 0 & 1 & 1 & 0 & 1 & 0 & 1 & 1 & 1 \\
\hline
\end{tabular}

Fig. 6. Schematic diagram of mutation operation.

- Finally, two offspring are produced as new chromosomes for the next genetic operation.

(6) Mutation operator

In this paper, the method of reverse mutation is used. First, two mutation points are randomly determined in the parent individuals, and then the gene positions between the two mutation points are inserted in reverse order. As shown in Figure 6, the parent individual is [011010111], the positions of the reverse order points are determined to be 4 and 8, then the mutated individual is [011110101].

(7) Termination condition

This article limits the maximum number of iterations to $T_{\max }=200$. The selection of genetic algorithm parameters in this paper is mainly based on previous algorithm design experiences and many accumulated experiments, including the number of genetic iterations, population numbers, selection operators, crossover operators, and mutation operators.

\section{Experiments and method verification}

When solving and optimizing the maintenance decisionmaking model considering the safety overhaul of fully mechanized mining equipment group, the "three machines and one frame"? in a coal mining company's working face refers to a series connection consisting of coal mining machine, hydraulic support, scraper conveyor and belt conveyor. The experiment method is to take the basic data
Table 1. Weibull parameter table of each equipment.

\begin{tabular}{lll}
\hline & $\begin{array}{l}\text { Size } \\
\text { parameter a }\end{array}$ & $\begin{array}{l}\text { Shape } \\
\text { parameter b }\end{array}$ \\
\hline Shearer & 3.89 & 5.15 \\
Hydraulic support & 4.93 & 5.5 \\
Scraper conveyor & 4.53 & 4.9 \\
Belt conveyor & 4.73 & 4.85 \\
\hline
\end{tabular}

Table 2. Maintenance schedule of each equipment.

\begin{tabular}{llll}
\hline & $\begin{array}{l}\text { Small } \\
\text { scale } \\
\text { repair/ } \\
\text { hour }\end{array}$ & $\begin{array}{l}\text { Medium } \\
\text { scale } \\
\text { repair/ } \\
\text { hour }\end{array}$ & $\begin{array}{l}\text { Item } \\
\text { repair/ } \\
\text { hour }\end{array}$ \\
\hline Shearer & 2 & 5 & 7 \\
Hydraulic support & 2 & 4 & 13 \\
Scraper conveyor & 1.5 & 3 & 9 \\
Belt conveyor & 2 & 2.5 & 9 \\
\hline
\end{tabular}

of the production system as an example, use MATLAB R2014b for genet-ic algorithm design, and perform experimental verification, and get experimental results.

In this paper, when the model is optimized and solved, each device's basic parameters are first imported. The Weibull parameter table is shown in Table 1 , and the maintenance time is shown in Table 2. The maintenance plan period is set at 12 months, and the maintenance interval is di-vided into equal intervals monthly. The final maintenance decision-making arrangement is de-termined by determining each device's opportunity maintenance activities in each interval. "Three shifts and one shift" are implemented for production work every day. According to Cui and Ma [16], the coal mine safety is evaluated, and it is finally determined that the coal mine safety requirement should not be less than $80 \%$. During the maintenance plan period, the system's continuous production unit time production benefit $V$ is 11,352 yuan.

- It is stipulated that the accelerated degradation factors for minor repairs, intermediate repairs and item repairs of fully mechanized mining equipment groups are: $\alpha=0.98^{\mathrm{Mnum}}, \alpha=0.97^{\mathrm{Mnum}}, \alpha=0.96^{\mathrm{Mnum}}$, Mnum is reduced by 1 for each maintenance. The performance recovery factors of intermediate repair and item repair are: $\beta=0.97^{\text {Inum }}, \beta=0.98^{\text {Inum }}$, Inum increases by 1 every time it is maintained. Mnum and Inum are the change value set in the formula.

- The same equipment and the same maintenance method have the same maintenance cost each time, namely $C_{d}=1500$ (Fixed shutdown loss per hour of downtime), $C_{i, M}=8000, C_{i, I}=18000, C_{i, R}=30000$.

- When the equipment is inspected and repaired safely, the cost of inspection only if there is no safety hazard is $C_{f}=4880$, The probability of a potential safety hazard is $p_{m}\left(t_{i}\right)=15 \%$ The probability that there is no safety hazard is $p_{n}\left(t_{j}\right)=85 \%$. 
- Within 365 days after certain equipment was repaired and started to operate, it completed 1 working face mining task, carried out 1 relocation and inversion. It took 30 days for the first move and reversion, and it took 212 days to complete the first fully mechanized mining face.

- When solving the genetic algorithm, the population size is set to 100, the maximum number of iterations is 200 the generation gap is 0.8 the selection probability is 0.8 and the $\mathrm{mu}$ - tation rate is 0.1 .

- The maintenance thresholds are respectively $0.3,0.5$ and 0.6 .

\subsection{Analysis of maintenance decision results}

In the maintenance plan period, consider the economic relevance and structural relevance to conducting maintenance decision optimization research on the coal miner's comprehensive min-ing equipment group. Through the iterative operation of the genetic algorithm, the results of each equipment operation are obtained. Figure 7 is the maintenance decision effect diagram of the fully mechanized mining equipment group during the entire maintenance plan period; Figure 8 is the maintenance effect diagram of each equipment.

From the analysis of Figures 7 and 8, it can be seen that: first, the fully mechanized coal mining equipment group completed the mining task of a certain working face in the first 212 days; then it took 30 days to move the equipment and overhaul the task in the process, So the equipment failure rate is restored to 0.2 . It can also be seen from Figure 7 and Figure 8 that the failure rate of each equipment did not reach the maintenance threshold on the 90th, 181, and 334 days, but maintenance activities were carried out. Potential failures are discovered during the safety inspection of the comprehensive mining equipment group, so it is a maintenance ac-tivity generated by the safety inspection.

In the maintenance process, the opportunity maintenance strategy is adopted to keep the equip-ment failure rate at a low level, to ensure that the entire system can reduce downtime for a long time to maintain smooth operation. The maintenance decision arrangement is shown in Table 3.

The analysis in Table 3 shows when the minor repairs, intermediate repairs, and item repairs of different equipment are completed. The maintenance decisionmaking arrangement can provide decision-making guidance and reference for equipment maintenance.

\subsection{Compared with traditional methods}

(1) Comparison of maintenance strategies

The genetic algorithm is used to iteratively optimize the maintenance decision model considering the safety overhaul of the fully mechanized mining equipment group to obtain the lowest maintenance decision cost. Through the iterative optimization of the maintenance decision in Figure 9, the objective function value is reduced from 2.92 million to 1.63 million.

The convergence rate of the program operation is shown in Figure 9. When the number of iterations is 133, the total maintenance cost reaches about 1.63 million yuan. It reaches a stable state, indicating that it is the optimal maintenance cost for maintenance decision-making arrangements. To consider the impact of opportunistic maintenance strategies on the maintenance decisionmaking effect of coal mine enterprises, this paper uses the same data to adopt regular maintenance strategies commonly used in equipment maintenance of coal mine enterprises to conduct maintenance decision optimization research fully mechanized mining equipment groups. The final optimization result has a total maintenance cost of

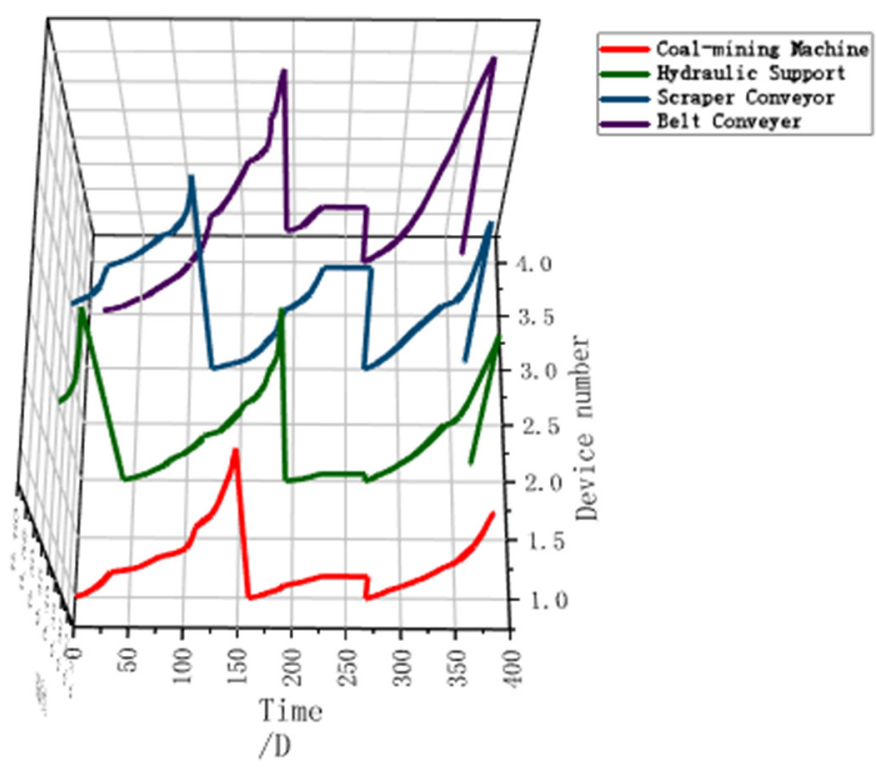

Fig. 7. The effect diagram of the opportunity maintenance decision of the fully mechanized mining equipment group.

Table 3. Maintenance decision-making schedule of coal mine comprehensive mining equipment group.

\begin{tabular}{llll}
\hline Mining equipment group & Minor repair/Day & Medium level repair/Day & Item repair \\
\hline Shearer & $40,90,121,334,364$ & - & 160 \\
Hydraulic support & $121,160,181,334$ & 364 & 40,193 \\
Scraper conveyor & $40,90,193,334$ & 364 & 121 \\
Belt conveyor & $121,160,181,334$ & 193,364 & - \\
\hline
\end{tabular}




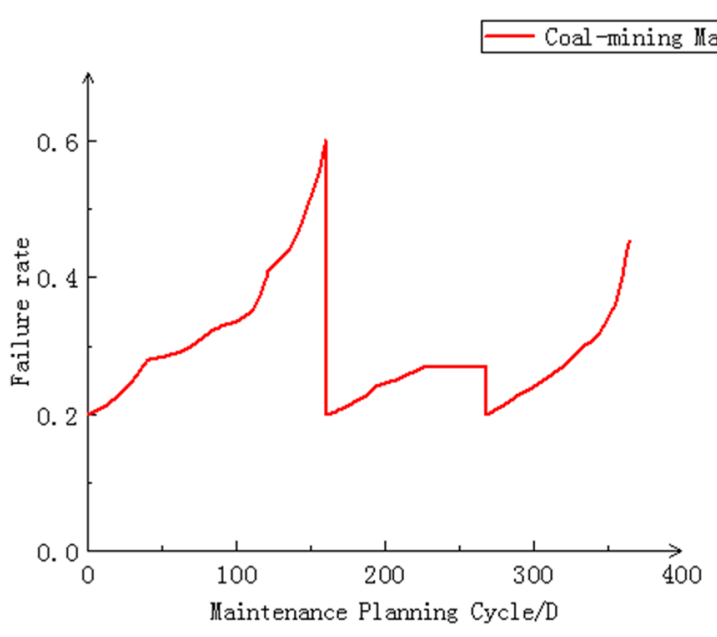

(a)

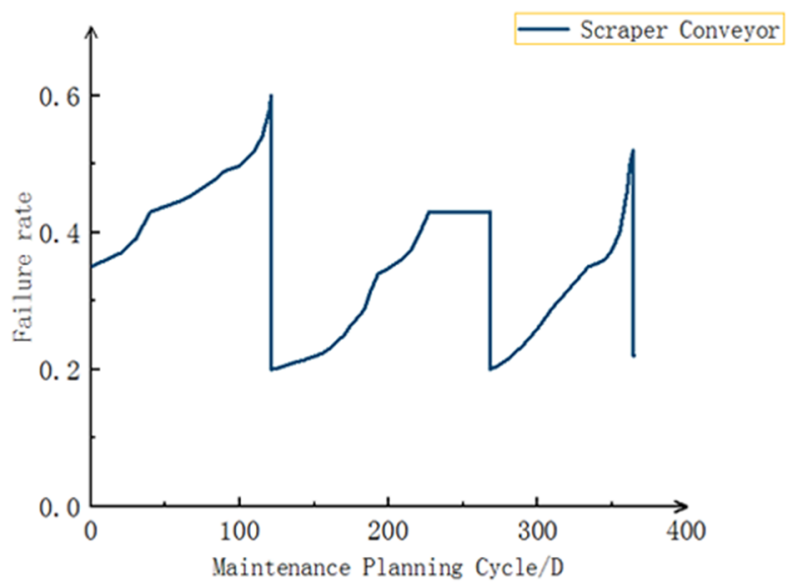

(c)

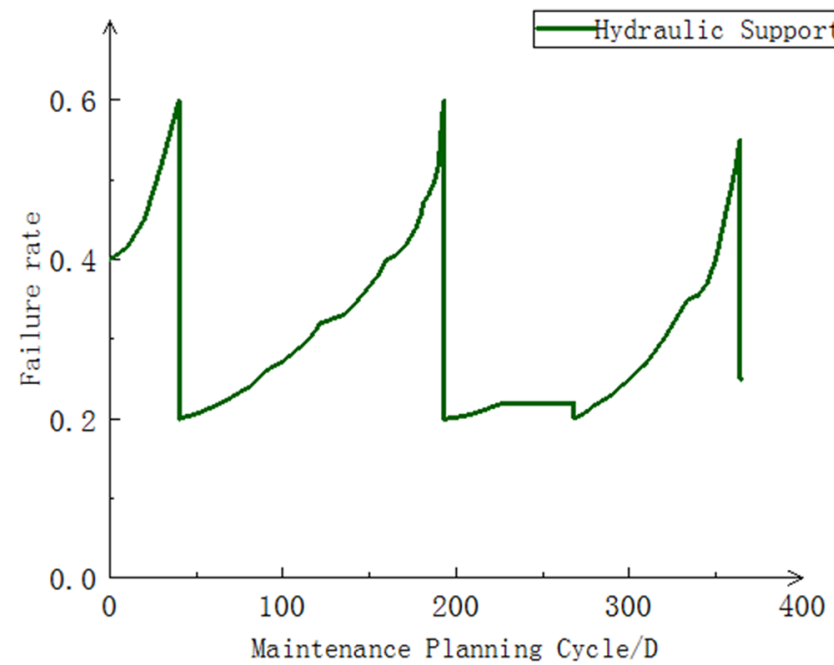

(b)

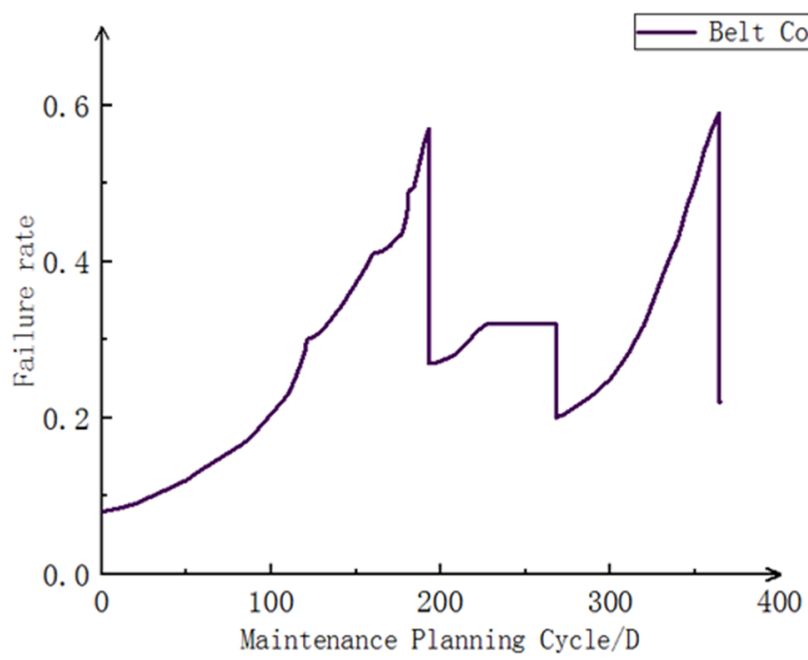

(d)

Fig. 8. Maintenance effect diagram of each equipment. (a) Effect drawing of shearer maintenance decision. (b) Hydraulic support maintenance decision effect diagram. (c) Effect drawing of maintenance decision for scraper conveyor. (d) Effect drawing of maintenance decision for belt conveyor.

2.542 million yuan as shown in Table 4. Compared with the regular maintenance strategy, the maintenance cost of this paper's opportunistic maintenance strategy can be reduced by $35.91 \%$. Therefore, it is proved that applying the opportunistic maintenance to the maintenance of the fully mechanized coal mining equipment group can better reduce the maintenance cost.

\section{(2) Algorithm comparison}

In this paper, the genetic algorithm is used to make maintenance decisions for fully mechanized mining equipment group and compare with existing research methods. The current research paper [17] uses a heuristic algorithm to optimize the maintenance decision model. This paper uses the five heuristic rules and the sum of different weights established by it as the evaluation index for the maintenance decision, and the maintenance is given priority. When comparing algorithms, the genetic algorithm's maintenance thresholds, and this method are both $0.3,0.5$, and 0.6 .

As shown in Table 5, the optimization results of maintenance decision-making are compared from two aspects. It is evident that the genetic algorithm in this paper reduces the maintenance cost of 1.266 million yuan compared with the heuristic algorithm, and the number of maintenances is also reduced from 9 to 6 . Genetic algorithm optimization has the lowest maintenance cost and maintenance times. Therefore, applying a genetic algorithm to optimizing maintenance decision-making of fully mechanized mining equipment groups play a great role compared with a heuristic algorithm. 


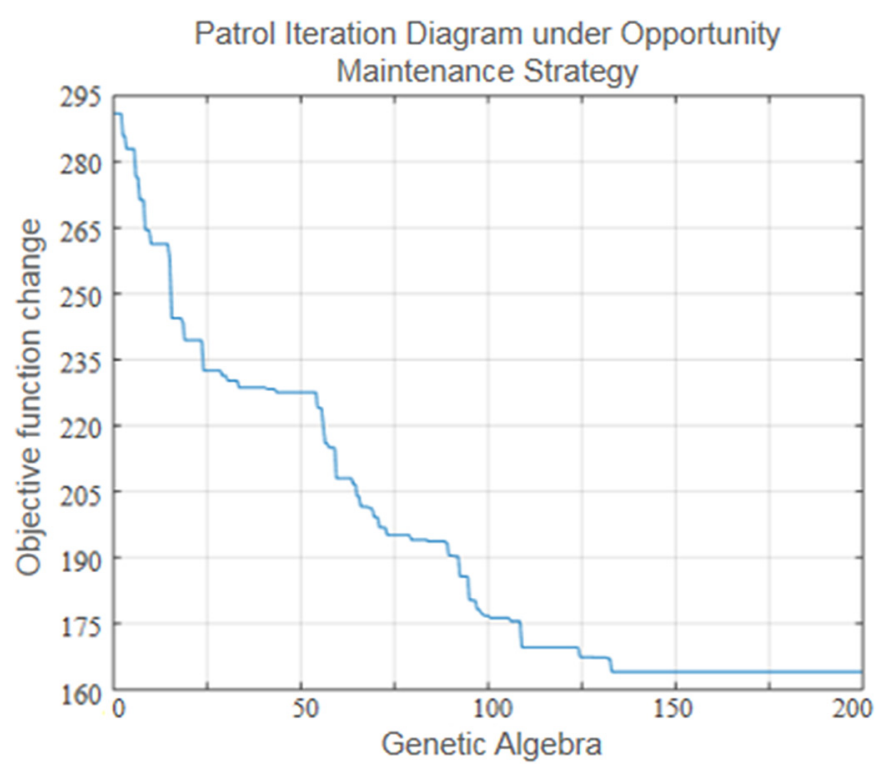

Fig. 9. Optimizing iterative graphs considering safety inspection and intenance decision-making.

Table 4. Maintenance strategy comparison table.

\begin{tabular}{lll}
\hline & $\begin{array}{l}\text { Regular } \\
\text { maintenance } \\
\text { strategy }\end{array}$ & $\begin{array}{l}\text { Opportunity } \\
\text { maintenance } \\
\text { strategy }\end{array}$ \\
\hline $\begin{array}{l}\text { Maintenance } \\
\text { cost/10,000 yuan }\end{array}$ & 254.2 & 163 \\
\hline
\end{tabular}

Table 5. Algorithm comparison table.

\begin{tabular}{lll}
\hline & $\begin{array}{l}\text { heuristic } \\
\text { algorithm }\end{array}$ & $\begin{array}{l}\text { genetic } \\
\text { algorithm }\end{array}$ \\
\hline Cost/ 10,000 Yuan & 289.6 & 163 \\
Number of repairs & 9 & 6 \\
\hline
\end{tabular}

\section{Conclusion}

This article first describes the maintenance decisionmaking issues, analyzes the importance of equipment safety issues to coal production, and establishes a maintenance decision-making model that considers the safety overhaul of fully mechanized mining equipment groups based on the harsh underground environment and high safety requirements of the coal mine; The design of coding, fitness function, selection, crossover, mutation operator, and parameter selection has carried out specific genetic algorithm design for the model. Finally, the model's rationality in this paper is verified by experiments, which proves that business decision-makers can use the research method in this paper. Provide guidance and reference for maintenance decision-making arrangements. And comparing the maintenance cost optimization value of the opportunistic maintenance strategy is compared with the regular maintenance strategy, the opportunistic maintenance cost is relatively reduced by $35.91 \%$. Therefore, it shows that this article adopts opportunistic maintenance and genetic algorithm to optimize the maintenance cost more reasonably and effectively. Comparing the genetic algorithm with the heuristic algorithm, the genetic algorithm cost optimization value is reduced by $43.72 \%$, and the maintenance frequency is reduced from 9 to 6 . Therefore, it shows that the optimization of maintenance cost by opportunistic maintenance and genetic algorithm in this chapter is more reasonable and effective.

\section{References}

1. L. Yibo, B. Yunhu, H. Jianguo, Discussion on developing fully mechanized top coal caving mining and existing problems and counter measures, Saf. Coal Mines 42, 160-162 (2011)

2. J. Jie, Research on the reliability of coal mine machinery and equipment, J. Xi'an Univ. Sci. Technol. 34, 75-80 (2014)

3. X. Kai, Z. Bin, F. Huijuan et al., Demand analysis of auxiliary decision-making system for vehicle equipment maintenance task allocation based on RCM, J. Military Transp. Inst. 33-37 (2019)

4. Y. Yuan, L. Fang, L. Zhenyu, Z. Baohua, Group optimization of system preventive maintenance tasks based on correlation, J. Wuhan Univ. (Engineering Edition) 45, 539-544 (2012)

5. L. Lin, B. Luo, S.S. Zhong, Development and application of maintenance decision-making support system for aircraft fleet, Adv. Eng. Softw. 114, 192-207 (2017)

6. A.S. Eruguz, T. Tan, G.-J. van Houtum, Optimizing usage and maintenance decisions fork-out-of-n systems of moving assets, Naval Res. Logistics 64, 418-434 (2017)

7. H. Yong, W. Hong, Y. Guojun, W. Hongyu, The optimal model of phased preventive maintenance for EMU components based on multi-objective decision-making, Ind. Eng. Manag. 23, 50-56 (2018)

8. F. Xiaogang, Discussion on several issues related to regulations and standards in coal industry safety, Coal Sci. Technol. 45, 72-75 (2017)

9. T. Zhanglu, C. Xiaoci, Research on coal mine safety hazard management based on text mining, China Work Saf. Sci. Technol. 16, 43-48 (2020)

10. C. Xiangang, G. Yurong, L. Xuan, L. Yinan, Z. Shunan, Research on optimization of maintenance decision-making for fully mechanized coal mining equipment group considering opportunity maintenance, Coal Eng. 52, 164-169 (2020)

11. S. Wei, Group screening method in reliability test of coal mine machinery products, Coal Mine Mach. 34, 70-71 (2013) 
12. Z. Xiaowen, J. Zuhua, H. Jiawen, Opportunistic maintenance of multi-stage bottleneck series/parallel system based on theory of constraints, J. Harbin Eng. Univ. 37, 1275 (2016)

13. H. Canh Vu, P. Do, A. Barros, C. Bérenguer, Maintenance grouping strategy for multi-component systems with dynamic contexts, Reliab. Eng. Syst. Safety 132 (2014)

14. W. Fuqiang, Research on the establishment of quotas for overhaul of fully mechanized coal mining equipment, Coal Eng. 51, 116-119 (2019)
15. C. Yan, W. Zongxian, Differential evolution algorithm based on multi-criteria optimization strategy, J. Shandong Univ. Sci. Technol. (Natural Science Edition) 35, 102-108 (2016)

16. C. Tiejun, M. Yundong, Research on coal mine safety situation differentiation method based on factor space, Syst. Eng. Theory Practice 35, 2891-2897 (2015)

17. M.E. Villalba Matamoros, M. Kumra, J. Central South Univ. 26, 3126-3139 (2016)

Cite this article as: Xian Gang Cao, Meng Yuan Zhang, Yu Rong Gong, Xiao Lin Jia, Rui Yuan Zhang, Maintenance decision method considering inspection of mining equipment, Int. J. Metrol. Qual. Eng. 12, 21 (2021) 